\title{
The Role of Transcriptomic Biomarkers of Endometrial Receptivity in Personalized Embryo Transfer for Patients with Repeated Implantation Failure
}

\author{
Aihua $\mathrm{He}$ \\ Department of Reproductive Medicine, Xiangya Hospital, Central South University \\ Yangyun Zou \\ Department of Clinical Research, Yikon Genomics Company, Ltd.

\section{Cheng Wan} \\ Department of Clinical Research, Yikon Genomics Company, Ltd.

\section{Jing Zhao}

Department of Reproductive Medicine, Xiangya Hospital, Central South University Qiong Zhang

Department of Reproductive Medicine, Xiangya Hospital, Central South University

\section{Zhongyuan Yao}

Department of Reproductive Medicine, Xiangya Hospital, Central South University

\section{Fen Tian}

Department of Reproductive Medicine, Xiangya Hospital, Central South University

\section{Hong Wu}

Department of ENT, Xiangya Hospital, Central South University

\section{Xi Huang}

Department of Reproductive Medicine, Xiangya Hospital, Central South University Jing Fu

Department of Reproductive Medicine, Xiangya Hospital, Central South University

Chunxu Hu

Department of Clinical Research, Yikon Genomics Company, Ltd.

\section{Yue Sun}

Department of Clinical Research, Yikon Genomics Company, Ltd.

\section{Lan Xiao}

Department of Reproductive Medicine, Xiangya Hospital, Central South University Tianli Yang

Department of Reproductive Medicine, Xiangya Hospital, Central South University Zhaojuan Hou

Department of Reproductive Medicine, Xiangya Hospital, Central South University 


\section{Xin Dong}

Department of Clinical Research, Yikon Genomics Company, Ltd.

\section{Sijia Lu}

Department of Clinical Research, Yikon Genomics Company, Ltd.

\section{Yanping Li ( $\boldsymbol{\nabla}$ liyanp@csu.edu.cn )}

Department of Reproductive Medicine, Xiangya Hospital, Central South University. Clinical Research Center for Women's Reproductive Health in Hunan Province https://orcid.org/0000-0002-4193-7321

\section{Research}

Keywords: Endometrial receptivity, window of implantation, biomarkers, RNA-Seq, repeated implantation failure, personalized embryo transfer

Posted Date: December 16th, 2020

DOI: https://doi.org/10.21203/rs.3.rs-126797/v1

License: (c) (i) This work is licensed under a Creative Commons Attribution 4.0 International License. Read Full License 
1 The role of transcriptomic biomarkers of endometrial receptivity in 2 personalized embryo transfer for patients with repeated implantation 3 failure

4

Aihua $\mathrm{He}^{1,2}$, Yangyun Zou ${ }^{3}$, Cheng $\mathrm{Wan}^{3}$, Jing Zhao ${ }^{1,2}$, Qiong Zhang ${ }^{1,2}$, Zhongyuan Yao ${ }^{1,2}$, Fen $\mathrm{Tian}^{1,2}$, Hong $\mathrm{Wu}^{4,5}$, Xi Huang ${ }^{1,2}$, Jing $\mathrm{Fu}^{1,2}$, Chunxu $\mathrm{Hu}^{3}$, Yue Sun ${ }^{3}$, Lan Xiao ${ }^{1,2}$, Tianli Yang ${ }^{1,2}$, Zhaojuan $\mathrm{Hou}^{1,2}$, Xin Dong ${ }^{3}$, Sijia Lu ${ }^{3 *}$, Yanping Lij ${ }^{1,2 *}$

${ }^{1}$ Department of Reproductive Medicine, Xiangya Hospital, Central South University, Changsha, Hunan, China

${ }^{2}$ Clinical Research Center for Women's Reproductive Health in Hunan Province, Changsha, Hunan, China

${ }^{3}$ Department of Clinical Research, Yikon Genomics Company, Ltd., Suzhou, Jiangsu, China.

${ }^{4}$ Department of ENT, Xiangya Hospital, Central South University, Changsha, Hunan, China

${ }^{5}$ Key Laboratory of Otolaryngology in Hunan Province, Changsha, Hunan, China

\section{*Correspondence}

Yanping Li, Department of Reproductive Medicine, Xiangya Hospital, 87 Xiangya Road, Changsha City, Hunan Province, 410000 , China.

E-mail: liyanp@csu.edu.cn.

Sijia Lu, Department of Clinical Research, Yikon Genomics Company, Ltd., \#301, Building A3, No. 218, Xinghu Street, Suzhou, Jiangsu. 215123, China.

E-mail: lusijia@yikongenomics.com 


\begin{abstract}
Background: Window of implantation (WOI) displacement was known as one of endometrial origin leading to embryo implantation failure, especially for repeated implantation failure (RIF). A accurately prediction tool of endometrial receptivity (ER) is extraordinary needed to precisely guide the successful embryo implantation. We aimed to establish an RNA-seq based endometrial receptivity test tool (rsERT) using transcriptomic biomarkers, and to evaluate the benefit of personalized embryo transfer ( $\mathrm{pET}$ ) guided by this tool in patients with repeated implantation failure (RIF).
\end{abstract}

Methods: Two-phase strategy including tool establishment with retrospective data and benefit evaluation with prospective, nonrandomized controlled trial. In the first phase, the rSERT was established by sequencing and analyzing the RNA of endometrial tissues from 50 infertile patients with normal window of implantation (WOI) timing. In the second phase, 142 patients with RIF were recruited and grouped by patient self-selection (experimental group, $n=56$; control group, $\mathrm{n}=86$ ). $\mathrm{pET}$ guided by rSERT in the experimental group, and conventional $\mathrm{ET}$ in the control group.

Results: The rsERT, comprising 175 biomarker genes, showed an average accuracy of $98.4 \%$ by using 10 -fold cross-validation. IPR of experimental group (50.0\%) was significantly improved compared to that $(23.7 \%)$ of control group (RR, $2.107 ; 95 \% \mathrm{Cl}, 1.159$ to $3.830 ; P=0.017$ ) when transferring day 3 embryos. Although not statistically different, IPR of experimental group (63.6\%) was still 20 percentage points higher than that $(40.7 \%)$ of control group (RR, 1.562; $95 \% \mathrm{Cl}, 0.898$ to $2.718 ; P=0.111$ ) when transferring blastocyst. Regression analysis can precisely predict the optimal WOI time by using all samples as training dataset $\left(R^{2}=0.92\right)$.

Conclusions: The rsERT was developed to accurately predict WOI period and significantly improve pregnancy outcomes of patients with RIF, indicating the clinical potential of rSERT-guided pET. Optimization of the model made it possible to predict the optimal WOI by one-point sampling.

Trial registration: Chinese Clinical Trial Registry: ChiCTR-DDD-17013375. Registered 14 November 2017, http://www.chictr.org.cn/index.aspx

\title{
Keywords
}

Endometrial receptivity, window of implantation, biomarkers, RNA-Seq, repeated implantation failure, personalized embryo transfer

\section{Background}

A successful pregnancy depends upon successful human embryo implantation, which requires a receptive endometrium, a normal and functional embryo at the blastocyst developmental stage and a synchronized dialogue between the maternal and embryonic tissues ${ }^{[1]}$. Inadequate endometrial receptivity (ER), defined as the ability of the endometrium to accept and accommodate a nascent embryo, is responsible for approximately two-thirds of implantation failures ${ }^{[2]}$. This period of receptivity is known as the "window of implantation" (WOI) ${ }^{[3]}$, which usually occurs on cycle days 19-24 of the menstrual cycle ${ }^{[4]}$, on day 7 after $\mathrm{LH}$ surge( $\left.\mathrm{LH}+7\right)$ in the natural cycle or on day 5 after progesterone administration $(P+5)$ in the artificial cycle $e^{[2}, \underline{5}$. Traditionally, the WOI was thought to be quite wide, but the optimal window has been shown to be much smaller, possibly lasting for only 2 days ${ }^{[6]}$. Delayed implantation at the extremes of the endometrial window can result in poor pregnancy outcomes ${ }^{[z]}$. Therefore, objective and accurate 
determination of the optimal WOI is crucial for improving the outcomes of pregnancy facilitated by assisted reproductive technology (ART).

The length of the WOI is not constant in all women and some present WOI displacement, which can delay, advance or narrow the WOI[8]. This can contribute to embryo-endometrial asynchrony, which usually results in implantation failure or even repeated implantation failure (RIF) ${ }^{[9]}$ defined by some as a minimum of two IVF or frozen cycle failures in which at least four morphologically high-quality cleavage embryos or two high-quality blastocysts were transferred ${ }^{[10]}$. In IVF-ET, the incidence of RIF is as high as $5-10 \%[11]$ and about $60 \%$ of RIF can be attributed to abnormal maternal ER which presents as a displacement and/or pathological disruption of the WOI ${ }^{[12]}$. Displacement of WOI present in 1 of 4 patients with RIF[13]. Thus, the accurate identification of the optimal WOI in patients with RIF by an effective diagnostic tool and subsequent personalized embryo transfer (pET) to restore synchronicity of embryonic and endometrial development, can allow for successful embryo implantation ${ }^{[14]}$.

The study of ER dates back to the 1950s, when Noyes et al established classical histological criteria for the evaluation of ER by analyzing endometrial staging and receptivity status ${ }^{[15]}$. And then, many studies have sought to define a healthy WOI by various ER markers through ultrasonography $\underline{[16-20]}$, morphology ${ }^{[21]}$, and molecular biology[22-25]. However, the objectivity, accuracy, reproducibility and functional relevance of these studies have been questioned $[26-28]$

As new high-throughput "omics" studies have emerged, the endometrial transcriptome has provided a deeper understanding of $\mathrm{ER}^{[\underline{20}]}$, and the feasibility of a molecular diagnostic tool that can identify a receptive endometrium based on a specific transcriptomic signature in different stages of the endometrial cycle has been demonstrated ${ }^{[30-32]}$. For example, an endometrial receptivity array (ERA) based on microarray technology coupled to a computational predictor was able to identify the WOI by predicting the receptivity status of endometrial biopsy samples objectively and accurately. ERA contains 238 genes which were screened from the differential gene expression profiles between pre-receptive and receptive status $\underline{[33]}$. ERA is more accurate than histological methods and results were reproducible in the same patients $29-40$ months after the first test ${ }^{[34]}$. Moreover, these studies have demonstrated the clinical value of ERA in patients with RIF for guiding $\mathrm{pET}^{[35]}$. Recent multicenter randomized clinical trial has indicated the potential of ERA test in the diagnosis of endometrial factors in the work-up of the infertile couple at the first appointment ${ }^{[36]}$. Before performing $\mathrm{PET}$, infertile patients underwent one or two endometrial biopsies for the ERA test to accurately determine the receptive period. Their pregnancy, implantation and cumulative live birth rates can be statistically significantly improved by $\mathrm{PET}$ guided by the ERA test. This provides a basis for further development of ER molecular diagnostic tools for reproductive clinic through endometrial transcriptome research.

RNA-Seq is one of the new generation high-throughput sequencing techniques used in transcriptomics research. Compared with conventional microarrays, RNA-Seq has the benefits of ultra-high sensitivity, dynamic range, more accurate quantification, and whole-transcriptome analysis which would allow identification of the ER differentially expressed genes(DEGs) from an unrestricted range of genes ${ }^{[37]}$. ER diagnostic methods can be further improved by transcriptomic analysis of the endometrial receptivity using RNA-seq.

To improve molecular diagnostic tool for ER, we improved experimental design, endometrial biopsy sampling time, and combined with machine learning algorithm to construct a novel RNA-seq based endometrial receptivity test ( $r S E R T)$ consisted of ER-specific marker genes, and to 
investigate whether $\mathrm{pET}$ guided by rSERT can improve pregnancy outcomes in patients with RIF. At the same time, we try to optimize rsERT's ability to predict the optimal WOI with hour precision, so that one-point sampling detection is possible.

\section{Methods}

\section{Study design and participants}

This study was conducted at the Department for Reproductive Medicine at Xiangya Hospital in Changsha, Hunan, People's Republic of China. The study was approved by the Reproductive Medicine Ethics Committee of Xiangya Hospital, and was registered in the Chinese Clinical Trial Registry (registration no.: ChiCTR-DDD-17013375).

All patients were undergoing IVF between November 2017 and July 2019. This study describes two separate phases.

In the first phase, from November 2017 to December 2018, participants were recruited to identify DEGs among pre-receptive, receptive and post-receptive endometrium and to build the rSERT. To limit interference from confounding variables affecting ER, the inclusion criteria for IVF patients were as follows: $20-39$ years of age; body mass index $(B M I)=18-25 \mathrm{~kg} / \mathrm{m}^{2}$; secondary infertility with a history of a intrauterine pregnancy/pregnancies and undergoing the first IVF cycle due to tubal factors; primary infertility undergoing the first IVF cycle due to male factors; a regular menstrual cycle length (25-35 days) with spontaneous ovulation; normal ovarian reserve (baseline $\mathrm{FSH}<10 \mathrm{mlU} / \mathrm{mL}$, antimullerian hormone $>1.5 \mathrm{ng} / \mathrm{ml}$, antral follicle count $>5$ ); able to be followed up to assess the pregnancy outcome, and successful intrauterine pregnancy after the first embryo transfer (ET). The intrauterine pregnancy was defined as the presence of a gestational sac with or without fetal heart activity in the uterine cavity as evaluated by ultrasound 4-5 weeks after ET. To establish the prediction tool, normal ER status was defined with successful intrauterine pregnancy.

In the second phase, from May 2018 to July 2019, participants were recruited to demonstrate the clinical impact of the rSERT in patients with RIF. This study was designed as a prospective, nonrandomized concurrent controlled trial. No reliable data were available at the trial design to allow for an accurate sample size calculation. Therefore, based on the results of the pre experiment, we used the assumption that the intrauterine pregnancy rate was $60 \%$ in the experimental group and $25 \%$ in the control group, respectively, and consider a two-sided $P$ value to be deemed statistically significant at $P<0.05$ and a power of $80 \%$. Considering the $10 \%$ loss of follow up rate, 33 subjects would be required in each group. The calculations of sample size were conducted by PASS software (Version 11.0). The inclusion criteria for patients with RIF were as follows: $20-39$ years of age; $\mathrm{BMI}=18-25 \mathrm{~kg} / \mathrm{m}^{2}$; and a history of RIF, which was defined as failure to achieve a clinical pregnancy after the transfer of at least 4 morphologically high-quality cleavage embryos or 2 high-quality blastocysts in a minimum of 2 fresh or frozen cycles (the criteria for good-quality embryos were as follows: (i) cleavage-stage embryos: $\geq 7$ blastomeres and $<20 \%$ fragments on Day 3 after fertilization ${ }^{[38,39]}$, (ii) Blastocysts: $\geq 3$ BB on Day 5 and Day 6, graded based on the Gardner system ${ }^{[40]}$. After providing informed choice consent, patients with RIF who chose to performed rSERT model to predict and guide PET were included in the experimental group and those who chose not to receive rsERT and underwent conventional ET directly were included in the control group.

The following exclusion criteria were applied: endometrial diseases (including intrauterine 
adhesions, endometrial polyps, endometritis, endometrial tuberculosis, endometrial hyperplasia, and a thin endometrium); hydrosalpinx without proximal tubal ligation; submucous myomas, intramural hysteromyomas, or adenomyomas protruding towards the uterine cavity; endometriosis (stages III-IV); uterine malformations; and other medical or surgical co-morbidities were identified by consulting medical records, physical examination, blood test, B-ultrasound and $\mathrm{X}$-ray examination.

All patients were followed up to assess pregnancy outcomes, as follows: grade and number of embryos transferred for all participants were recorded. Blood $\beta$-human chorionic gonadotropin ( $\beta$-HCG) was measured 12 days after embryo transfer, and the intrauterine pregnancy and number of gestational sacs were determined by ultrasound 28 days after transfer in BHCG-positive patients. Subsequently, all patients diagnosed with an intrauterine pregnancy were followed up until delivery. The last follow-up date was May 2020.

\section{Endometrial biopsy, sample collection and processing}

Written informed consent was obtained before sample collection. For patients included in the model construction phase, ultrasound was initiated from day 10 of the menstrual cycle preceding the IVF cycle to monitor ovulation. Blood LH levels were dynamically measured daily when the follicle diameter was $\geq 14 \mathrm{~mm}$. Patients continue to undergo daily ultrasound monitoring of ovulation until follicular discharge. Endometrial tissues were collected using an endometrial sampler (AiMu Medical Science \& Technology Co.; Liaoning; China) on days 5, 7, and $9(\mathrm{LH}+5, \mathrm{LH}+7$, and $\mathrm{LH}+9$, respectively) after the $\mathrm{LH}$ surge (denoted as $\mathrm{LH}+0)$.

For patients with RIF in experimental group with a natural cycle, the timing of endometrial tissue sampling was the same as in the modelling group above. For hormone replacement (HRT) cycles, estradiol administration started on the third day of the menstrual cycle, and progesterone supplementation were started after at least 12 days of oestrogen usage if the endometrium was $>7 \mathrm{~mm}$ and the endogenous $P$ serum level is close to zero. The day of starting progesterone supplementation considered as $P+0$, endometrial tissues were collected on days 3,5 , and 7 after progesterone supplementation (i.e., on days $\mathrm{P}+3, \mathrm{P}+5$, and $\mathrm{P}+7$, respectively).

In all cases the sampling was performed as follows. The cervix was cleansed with saline before sampling. The tip of the endometrial sampler was placed into the uterine fundus, and 5-10 $\mathrm{mm}^{3}$ of endometrial tissues were aspirated into the sampler. The collected endometrial tissues were immediately placed into $1.5 \mathrm{~mL}$ RNA-later buffer (AM7020; Thermo Fisher Scientific, Waltham, MA, USA) for RNA stabilization, sealed, and cryopreserved at $-20^{\circ} \mathrm{C}$. Sequencing analysis was carried out within 7 days after sampling.

\section{RNA extraction, library construction and sequencing}

Total RNA extraction was performed using the RNeasy Micro Kit (74004; Qiagen, Germantown, MD, USA) according to the instruction manual, followed by quantification with a Qubit HS RNA Kit (Q32855; Thermo Fisher Scientific, Waltham, MA, USA). Then, an RNA LabChip (Agilent Technologies, Santa Clara, CA, USA) was used in combination with an Agilent2100 Bioanalyzer (Agilent Technologies, Santa Clara, CA, USA) for integrity and quality control of the extracted total RNA. Samples with an RNA integrity number (RIN)> 7 were considered eligible for subsequent tests.

RNA reverse transcription was conducted using the MALBAC ${ }^{\circledR}$ Platinum Single Cell RNA 
Amplification Kit (KT110700796; Yikon Genomics, Suzhou, Jiangsu, China) according to the instruction manual. Both positive and negative control were included to ensure the experiments were conducted properly, which consist of 500ng high-quality human total RNA and ultrapure water, respectively. For this step, $1 \mu \mathrm{L}$ purified cDNA was reasonably diluted for detection on the Agilent 2100 Bioanalyzer High Sensitivity DNA Chip (Agilent Technologies, Santa Clara, CA, USA) according to the instruction manual. cDNA with a size of 1000-10000 bp was considered satisfying the quality control requirements.

Library construction was accomplished using the gene sequencing and library preparation kit (XY045; Yikon Genomics, Suzhou, Jiangsu, China) according to the instruction manual. After purification, the libraries were quantified using the Qubit dsDNA HS Assay Kit (Q32584; Invitrogen). Based on the results of Qubit quantitation, $10 \mathrm{ng}$ of the library was taken for each sample and mixed in equal proportion.

The mixed libraries were again subjected to the Qubit quantitation assay. Then, single-end sequencing was performed on the HiSeq 2500 platform (Illumina, San Diego, CA, USA) under relevant parameters. The read length was set to $140 \mathrm{bp}$. The volume of raw data was approximately $5 \mathrm{M}$ reads.

\section{Identification and functional annotation of the DEGs}

The raw sequencing reads were filtered to exclude low quality reads and alternative alignment with RNA-SeQC[41]. Qualified reads were mapped to the human reference genome (Ensembl primary assembly, version GRCh37) by using STAR ${ }^{[42]}$. The RNA expression level was estimated by FPKM ${ }^{[43]}$ (Fragments Per Kilobase Million) of each gene. Base-2 logarithmic transformation of FPKM was conducted for further analyses.

DEGs among the different ER conditions were identified by analysis of variance (ANOVA) with the following equation: $Y_{g i j k}=\mu_{g}+T_{g i}+S_{g j}+\varepsilon_{g i j k}$, where $\mu_{g}$ represents the mean expression level of gene $g ; T_{g i}$ is the gene-specific treatment effect referring to whether the patient had a natural cycle or was undergoing hormone replacement therapy when the endometrial tissue was obtained, $T_{g i} \sim\left(0, \sigma_{T_{g}}^{2}\right) ; S_{g j}$ is the gene-specific ER stage effect with three levels (pre-receptivity, receptivity, and post-receptivity), $S_{g i} \sim\left(0, \sigma_{S_{g}}^{2}\right)$; and $\varepsilon_{i j g k}$ is the gene-dependent residual error, $\varepsilon_{i j g k} \sim\left(0, \sigma_{\varepsilon_{g}}^{2}\right)$. The F-test was applied to statistically assess the equality of variances between $S_{j}$ and $\varepsilon_{i j k}$ for each gene, showing whether the gene is differentially expressed among the different ER stages. Because RNA-Seq analysis involves multiple statistical tests, the false discovery rate (FDR) was used to adjust the $p$-value (q-value) to provide statistical inference (Benjamini and Hochberg, 1995). Functional analysis of these DEGs was conducted by the DAVID tool based on Gene Ontology (GO)-based biological processes, cellular components and molecular functions and Kyoto Encyclopedia of Genes and Genomes (KEGG) pathways. 


\section{Candidate marker genes selection and predictive tool construction}

The samples from first phase were used as training dataset for prediction model construction of ER status. The cut-off q-value of $1 e-10$ was used to select the DEGs. The expression values of these DEGs were then inputted as features for the random forest machine learning method to train the pattern on three ER conditions (pre-receptivity, receptivity, and post-receptivity). The importance of each feature (gene expression) was calculated with the $R$ package random Forest by two measures (mean decrease accuracy and mean decrease Gini). The out-of-bag (OOB) error, mean accuracy from 10-fold cross-validation, and area under the receiver operating characteristic (ROC) curve (AUC) were calculated to evaluate the performance of the predictor.

For optimal WOI point prediction, we applied all samples as training dataset for model construction. With three-point sampling strategy and corresponding clinical outcome, numerical value with hour precision of optimal WOI in these training samples was defined. Specifically, if three samples from $\mathrm{LH}+5, \mathrm{LH}+7$ and $\mathrm{LH}+9$ in one patient were predicted as pre-receptivity, post-receptivity and post-receptivity, blastocyst(s) afterwards was/were transferred on LH+6 (day 3 cleavage embryo(s) transferred on $\mathrm{LH}+4$ ) and successfully implanted; In this case, the numerical hour value for these three samples was respectively $24 \mathrm{~h},-24 \mathrm{~h}$ and $-72 \mathrm{~h}$. There are some of such kind of different combinations, leading to $0 h, 24 h,-24 h, 48 h,-48 h, 72 h,-72 h, 96 h$ and $-96 h$. Random forest regression model (random Forest package) was therefore used to predict the optimal implantation point with hour precision. The R-square value of model fitting and 10-fold cross-validation approach were used to evaluate the predictive performance

\section{pET guided by the rsERT and outcome measures}

In the first frozen embryo transfer cycle after rsERT in the experimental group, pET was performed at the timing of optimal WOI predicted by rSERT which corresponds to the transfer of blastocysts, and day 3 cleavage embryos should be transferred 2 days earlier accordingly. Patients in control group directly underwent the conventional ET (i.e., transfers of frozen-thawed embryos or blastocysts were performed on the 5 or 7 days after the LH surge / after 3 or 5 days of progesterone supplementation).

Primary outcome measures were the intrauterine pregnancy rate (IPR). Secondary outcomes were live birth rate (LBR) and implantation rate (IR). We have adopted the following standardized definitions ${ }^{[44]}$. IPR: the number of patients with intrauterine pregnancy per embryo transfer cycles. LBR: the number of deliveries that resulted in at least one live birth per embryo transfer cycles. IR: the number of gestational sacs observed divided by the number of embryos transferred, single embryo transfer gestation sac counted as 1 only.

\section{Statistical analysis}

Continuous data subject to a normal distribution were expressed as the mean \pm SD, and were compared using independent-samples $t$-test. Continuous data subject to a skewed distribution were expressed as the median and inter-quartile range (IQR), and were compared using independent-samples Mann-Whitney $U$ test. Categorical data were expressed as counts and percentages, and were determined to be statistically significant using the chi-square test or Fisher's exact test. A two-side $P$-value equal or less than 0.05 was considered to be statistically significant. Statistical analysis was performed using IBM SPSS software (Version 23.0, IBM Corp.) 


\section{Results \\ Participants}

In the first phase, 71 participants were recruited and 21 patients who were not pregnant after the first embryo transfer were excluded, and 50 patients with successful intrauterine pregnancies were used to build rsERT model. Baseline clinical characteristics are shown in Table 1. In the second phase, of the 142 enrolled patients with RIF, 56 were assigned to experimental group and 86 to the control group by self-selection of patients. Baseline clinical characteristics were comparable among groups (Table 2). The percentage of blastocyst (day 5 or day 6) transferred in experimental group was significantly different from that in control group $(P=$ $0.013)$, while there was no significant difference in the percentage of high-quality day 3 cleavage embryos $(44 / 51,86.3 \%$ vs. $105 / 114,92.1 \%, P=0.376)$ and blastocysts $(17 / 39,43.6 \%$ vs. $19 / 44$, $43.2 \%, P=0.970$ ) between two groups. The two groups also showed no significant differences in the other lists. In the experimental group, 48 out of 56 patients with RIF received rsERT-guided $\mathrm{pET}$, except for 5 patients who had poor quality or collapsed freeze-thaw embryos and 3 patients who were lost to follow-up. All 86 patients with RIF in control group underwent conventional ET (Figure 1).

\section{Identification of DEGs among ER statuses}

To identify DEGs among pre-receptivity, receptivity, and post-receptivity stages that could then be used as biomarkers to predict ER, we compared the transcriptome of endometrial tissues collected on days $\mathrm{LH}+5, \mathrm{LH}+7$ and $\mathrm{LH}+9$ for patients with a natural cycle. In brief, we constructed 150 NGS libraries by using the total RNA extracted from endometrial biopsy samples of the 50 patients recruited in the first phase. An average of 6.7M raw reads were generated from 146 qualified libraries, with the mapping rate ranging from $18.8 \%$ to $86.5 \%$. Each library detected 14507 genes in average, and resulting a total of 3571 DEGs within the three different ER statuses, representing approximately $17 \%$ of all mapped genes.

Three well-defined groups were generated by the clustering analysis (Fig.2), in agreement with the timing of sampling. Functional analysis showed significant enrichment in embryo-endometrium interaction and embryonic implantation-related processes, such as protein transport (GO:001503), cell-cell adhesion (GO:0098609) and the oxidation-reduction process (GO:0055114) (in the biological process category); protein binding (GO:0005515) and protein homodimerization activity (GO:0042803) (in the molecular function category); and cell-cell adherens junction (GO:0005913), extracellular exosome (GO:0070062) and focal adhesion (GO:0005925) (in the cellular component category). KEGG pathways, including ECM-receptor interaction and signal transduction-related molecular function-like protein kinase binding, were alsoenriched in these DEGs (Supplementary information).

\section{Establishing and validating the ER predictive tool}

Next, we used the DEGs to construct a predictive model for the three ER conditions. The random forest algorithm was applied to train the model to recognize the pattern of RNA expression, resulting predictive markers containing 175 genes with mean decrease accuracy ranging from 3 to 5.43. Linear discriminant analysis (LDA) showed that the three ER conditions (pre-receptivity, receptivity, and post-receptivity) were distinctly classified by the expression 
pattern of these predictive markers. (Fig. 3A and Supplementary table). The average of 10-fold cross-validation was applied to assess the performance of the predictive model, resulting in a mean accuracy of $98.4 \%$ with $98.9 \%$ specificity and $97.8 \%$ sensitivity. ROC curve analysis of 100 random splits into a training set and a test set yielded an average area under the curve (AUC) of 99.1\% (Fig.3B).

\section{rsERT results in patients with RIF}

In the second phase study, a total of 168 NGS libraries were constructed for RNA-seq by using endometrial biopsy samples from patients in experimental group ( $n=56)$, with a qualification rate of $96.4 \%$ (162 of 168). The expression profile of selected markers was utilized to predict the ER status. The results indicated WOI displacement in 17 of 56 patients (30.4\%). Among them, advanced WOI occurred in 15 patients (15/17, 88.2\%), delayed WOI occurred in 2 patients $(2 / 17,11.8 \%)$. The WOI displacement was combined with narrowing in $10(10 / 17,58.8 \%)$ patients (WOI < $48 \mathrm{~h})$.

\section{Effect of rsERT guided pET on pregnancy outcomes in RIF}

Considering the significant difference in the percentage of transferred blastocysts between experimental group and control group, we compared the pregnancy outcomes of transferred day 3 cleavage embryos and blastocysts separately. The results showed that 26 out of 48 patients in experimental group and 59 out of 86 patients in control group had transferred day 3 embryos. IPR $(13 / 26,50 \%)$ and IR $(16 / 51,31.4 \%)$ in experimental group were significantly higher than IPR (14/59, $23.7 \%$ ) and IR (19/114, 16.7\%) in control group, respectively (RR, 2.107; 95\% Cl, 1.159 to 3.830; $P=0.017, \mathrm{RR}, 1.882 ; 95 \% \mathrm{Cl}, 1.057$ to $3.353 ; P=0.033)$. LBR $(11 / 26,42.3 \%)$ in experimental group was $20 \%$ higher than that $(13 / 59,22 \%)$ in control group, although not statistically $(R R, 1.92 ; 95 \%$ $\mathrm{Cl}, 0.995$ to 3.705; $P=0.056)$. In addition, 22 patients in experimental group and 27 patients in control group underwent blastocyst transplantation. IPR, LBR and IR (63.6\%, 59.1\% and 43.6\%) in the experimental group were all distinctly higher than that (40.7\%, 37\% and $27.3 \%)$ in the control group, but not statistically different $(\mathrm{RR}, 1.562 ; 95 \% \mathrm{Cl}, 0.898$ to $2.718 ; P=0.111, \mathrm{RR}, 1.595 ; 95 \%$ $\mathrm{Cl}, 0.874$ to $2.914 ; P=0.124, \mathrm{RR}, 1.598 ; 95 \% \mathrm{Cl}, 0.877$ to $2.913 ; P=0.120$ ) (Table 3 ).

\section{Optimal WOI estimation with hourly accuracy.}

Considering the endometrial invasion with three-point biopsy, we developed a random forest regression method to predict the optimal WOI point with one-point biopsy strategy. We therefore got endometrial biopsy samples of patients with successful intrauterine pregnancies from two-phase study for model training. Applying 10-fold cross-validation approach, each sample can be used as test data to be predicted once. Mean R-squared value of 0.92 was achieved, showing that $92 \%$ of the data fit the regression model. We found median of predicted WOI time in pre-receptivity, receptivity and post-receptivity samples are approximately 48 hours, 0 hour and -48 hours (Fig. 4A); Deviation points in Fig. 4A are mostly those WOI displacement samples, supporting the good performance of the model. Furthermore, by comparing predicted and expected value using 10 -fold cross-validation strategy, we found $94 \%$ and $85 \%$ of samples showed a deviation of less than 24 and 12 hours, respectively (Fig.4B, 4C). The method allows one-point biopsy to get the optimal WOI time, thus avoids second biopsy in the next cycle if the tested sample does not represent endometrial receptive status. Therefore, a process of $\mathrm{pET}$ 
guided by one-point sampling rsERT could be established, which would be more patient-friendly and cost-efficient.

\section{Discussion}

RIF is a highly challenging condition in ART with a complex etiology. Embryo quality and maternal endometrial factors are the main causes of RIF. At present, more and more attention has been paid to the effect of abnormal endometrial receptivity on RIF. WOI displacement disrupting the synchronicity of embryonic and endometrial development is one of the crucial causes of RIF. Therefore, in the current study, we aimed to use RNA-Seq to identify biomarkers for ER through transcriptome analysis and create a novel rSERT tool to accurately predict the optimal WOI and to improve the pregnancy outcomes of patients with RIF by pET guided by rsERT.

Through RNA-Seq, over 3000 DEGs were identified in our study, and GO annotation and KEGG pathway analyses showed marked enrichment in embryo implantation process-related functions and pathways. To date, several studies ${ }^{[45-47]}$ have revealed the transcriptome changes during different receptivity stages indicating the reliability and universality of DEGs as predictive biomarkers for ER. But unlike previous studies, we screened DEGs based on the following experimental methods and design advantages: Firstly, RNA-Seq used for sequencing analysis can provide a more precise and comprehensive view of transcriptome changes in the endometrial cycle than the conventional gene microarray; Secondly, comparing the sequencing data of the endometrial tissue samples $(\mathrm{LH}+5 / \mathrm{LH}+7 / \mathrm{LH}+9)$ among three different receptive status collected from the same patient at 48 hours interval during the same cycle can analyze more precisely the DEGs to identify marker genes for ER and their changing patterns during this narrow comparison time span which made it possible to accurately predict optimal WOI timing through one-point endometrial biopsy. Finally, the receptive period used as the contrast point was defined as day LH+7 determined by combined the blood LH surge with a subsequent intrauterine pregnancy, which is more reliable than the previous determination with $\mathrm{LH}$ surge alone, so that the obtained DEGs are more accurate. As result, 175 markers were selected from DEGs and established rsERT applied the random forest algorithm. The accuracy, specificity and sensitivity of rsERT for predicting the optimal WOI by 10 -fold cross-validation were $98.4 \%, 98.9 \%$ and $97.8 \%$, respectively.

In the second phase study, the rSERT was applied for patients with RIF, resulting WOI displacement rate of $30.4 \%$, which was similar to that in other studies $[\underline{48}, \underline{49}]$. However, unlike previous studies, most of the WOI displacement was advanced rather than delayed which may be related to the small sample size and regional population, and more clinical verification is needed. In addition to WOI displacement, 58.8\% of patients with RIF also exhibited narrowing of the WOI which allows shorter duration of WOI for embryo implantation, so it is particularly important to accurately predict the optimal WOI.

Subsequently, rsERT-guided pET was performed for patients in experimental group. Compared with control group, higher IPR, LBR and IR were obtained, especially for those who were transferred with day 3 cleavage embryos. The differences in IPR and IR were statistically significant, while LBR was 20 percentage points higher than that in control group (42.3\% vs. $22 \%$ ), although not statistically different. While IPR (63.6\% vs. $40.7 \%)$, LBR (59.1\% vs. 37\%) and IR $(43.6 \%$ vs. $27.3 \%)$ were not statistically different in the two groups when blastocysts were transferred. However, IPR and LBR in experimental group were higher than those in control group 
by more than 20 percentage points, and IR was also higher by 16 percentage points. This improvement was due to the restoration of the synchronicity of embryonic and endometrial development, rather than the influence of embryonic factors as shown by the lack of significant differences in the proportion of good-quality day 3 cleavage embryos and good-quality blastocysts transferred between two groups. These results demonstrate that use of the $p E T$ guided by rsERT significantly improved the pregnancy outcomes of patients with RIF.

After completing the construction and clinical validation of the rsERT model, another important objective of our study was to further optimize the detection model so that the optimal WOI period can be accurately predicted by a one-point sampling. Therefore, we developed an optimal WOI point estimation with hour precision, based on our three-point sampling prediction results and corresponding clinical outcomes. This method showed a high accuracy by retrospective analysis of patients with successful implantation. $94 \%$ of samples result in a deviation of less than 24 hours compared with observed WOI, only 6\% of samples showed larger deviation. In practice, there are a small proportion of women have longer duration of WOI than 48 hours ${ }^{[50]}$, which is in accordance with the result. Our data suggests the potential of developing a one-point sampling strategy, which could provide precise and cost-efficient rsERT. And in future work we will clinically validate this model.

Nonetheless, this study has several limitations. First, the sample size of this study is small and there is no application data of rsERT in infertile patients with conventional IVF. In order to clarify the clinical value of rsERT in infertile population with conventional IVF, we think it would be better to design a multicenter randomized controlled trial in the future. Second, according to the results of this study, $43.7 \%$ of patients with RIF still experienced implantation failure after pET guided by rsERT. Therefore, it can be inferred that in these patients, in addition to WOI displacement, there may also be ER pathological disruption. Another limitation of this study is that we cannot diagnose the pathological disruption of ER by evaluating the strength of WOI receptivity capacity. Our future work is to find marker genes representing WOI receptive capacity to establish a new ER detection model to diagnose pathological disruption of ER and to study the mechanism of ER marker genes, so as to provide theoretical basis for clinical treatment strategy. Of course, performing PGT to exclude embryonic factors is also an option to consider. Third, because of the invasive operation of endometrial sampling, non-invasive ERT is also our future research direction.

\section{Conclusions}

In summary, we built a novel rsERT that accurately predicts WOI period. It is consisted of ER-specific marker genes and screened by the combination of RNA-Seq and machine learning. rSERT-guided pET significantly improved pregnancy outcomes of patients with RIF, indicating the clinical potential of rsERT-guided pET. By optimizing rSERT's ability to predict the optimal WOI with hour precision, it made one-point sampling detection possible.

\section{Abbreviations}

ER: endometrial receptivity; WOI: window of implantation; RNA-seq: RNA sequencing; rsERT: RNA-Seq based endometrial receptivity test; ET: embryo transfer; pET: personalized embryo transfer; RIF: repeated implantation failure; ART: assisted reproductive technology; IVF: in vitro fertilization; IVF-ET: in vitro fertilization and embryo transfer; DEGs: differentially expressed genes; BMI: body mass index; ERA: endometrial receptivity array; FSH: 


\section{Authors' contributions}

Study concept and design: YL, SL and AH. Acquisition of data: AH, JZ, QZ, ZY, FT, HW, XH, JF, YS, TY. Collection of samples: $A H, X H, J F, L X, T Y, Z H$. Analysis and interpretation of data: $A H, Y Z, C W, C H, X D$. Drafting the manuscript: $A H, Y Z, C W, C H$. Supervision and critical revision of the manuscript for important intellectual content: $Y L, S L, A H$,

$\mathrm{YZ}, \mathrm{CW}$. All the authors read and approved the final manuscript.

\section{Author details}

$469{ }^{1}$ Department of Reproductive Medicine, Xiangya Hospital, Central South University, Changsha, Hunan, 410000,

470 China. ${ }^{2}$ Clinical Research Center for Women's Reproductive Health in Hunan Province, Changsha, Hunan, 410000,

China. ${ }^{3}$ Department of Clinical Research, Yikon Genomics Company, Ltd., Suzhou, Jiangsu, 215123, China. ${ }^{4}$ Department of ENT, Xiangya Hospital, Central South University, Changsha, Hunan, 410000, China. ${ }^{5}$ Key Laboratory of Otolaryngology in Hunan Province, Changsha, Hunan, 410000, China.

\section{Acknowledgements}

The authors also thanks to all the subjects who participated volunteered in the clinical trial and the medical team of the Department for Reproductive Medicine at Xiangya Hospital in China.

\section{Competing interests}

The authors declare that they have no competing interests.

\section{Availability of data and materials}

The primary data for this study is available from the corresponding author on reasonable request.

\section{Consent for publication}

All authors agreed the publication of this study.

\section{Ethics approval and consent to participate}

The study was approved by the Reproductive Medicine Ethics Committee of Xiangya Hospital. All participants enrolled in the study approved their participation studying and signing the informed consent.

\section{Funding}

This study was supported by grants from the National Natural Science Foundation of China (grant no. 8187061497), the Clinical Medical Technology Innovation Guide Project of Hunan Provincial Science and 


\section{References}

1. Norwitz ER, Schust DJ, Fisher SJ: Implantation and the survival of early pregnancy. N Engl J Med 2001, 345:1400-1408.

2. Messaoudi S, El Kasmi I, Bourdiec A, Crespo K, Bissonnette L, Le Saint C, Bissonnette F, Kadoch IJ: 15 years of transcriptomic analysis on endometrial receptivity: what have we learnt? Fertil Res Pract 2019, 5:9.

3. Psychoyos A: Uterine receptivity for nidation. Ann N Y Acad Sci 1986, 476:36-42.

4. Lessey BA: Assessment of endometrial receptivity. Fertil Steril 2011, 96:522-529.

5. Bergh PA, Navot D: The impact of embryonic development and endometrial maturity on the timing of implantation. Fertil Steril 1992, 58:537-542.

6. Prapas Y, Prapas N, Jones EE, Duleba AJ, Olive DL, Chatziparasidou A, Vlassis $\mathrm{G}$ : The window for embryo transfer in oocyte donation cycles depends on the duration of progesterone therapy. Hum Reprod 1998, 13:720-723.

7. Wilcox AJ, Baird DD, Weinberg CR: Time of implantation of the conceptus and loss of pregnancy. N Engl J Med 1999, 340:1796-1799.

8. Galliano D, Bellver J, Diaz-Garcia C, Simon C, Pellicer A: ART and uterine pathology: how relevant is the maternal side for implantation? Hum Reprod Update 2015, 21:13-38.

9. Teh WT, McBain J, Rogers P: What is the contribution of embryo-endometrial asynchrony to implantation failure? J Assist Reprod Genet 2016, 33:1419-1430.

10. Polanski LT, Baumgarten MN, Quenby S, Brosens J, Campbe11 BK, Raine-Fenning NJ: What exactly do we mean by ' recurrent implantation failure' ? A systematic review and opinion. Reprod Biomed Online 2014, 28:409-423.

11. Mak JSM, Chung CHS, Chung JPW, Kong GWS, Saravelos SH, Cheung LP, Li TC: The effect of endometrial scratch on natural-cycle cryopreserved embryo transfer outcomes: a randomized controlled study. Reprod Biomed Online 2017, 35:28-36.

12. Sebastian-Leon P, Garrido N, Remohi J, Pellicer A, Diaz-Gimeno P: Asynchronous and pathological windows of implantation: two causes of recurrent implantation failure. Hum Reprod 2018, 33:626-635.

13. Gomez E, Ruiz-Alonso M, Miravet J, Simon C: Human Endometrial Transcriptomics: Implications for Embryonic Implantation. Cold Spring Harb Perspect Med 2015, 5:a022996.

14. Ruiz-Alonso M, Galindo N, Pellicer A, Simon C: What a difference two days make: "personalized" embryo transfer (pET) paradigm: a case report and pilot study. Hum Reprod 2014, 29:1244-1247.

15. Noyes RW, Hertig AT, Rock J: Reprint of: Dating the Endometrial Biopsy. Fertil Steri1 2019, 112:e93-e115.

16. Zhao J, Zhang Q, Li Y: The effect of endometrial thickness and pattern measured by ultrasonography on pregnancy outcomes during IVF-ET cycles. Reprod Biol Endocrinol 2012, 10:100.

17. Zhao J, Zhang Q, Wang Y, Li Y: Endometrial pattern, thickness and growth in predicting pregnancy outcome following 3319 IVF cycle. Reprod Biomed Online 2014, 29:291-298. 
18. Hou Z, Zhang Q, Zhao J, Xu A, He A, Huang X, Xie S, Fu J, Xiao L, Li Y: Value of endometrial echo pattern transformation after hCG trigger in predicting IVF pregnancy outcome: a prospective cohort study. Reprod Biol Endocrinol 2019, $17: 74$.

19. Zhu L, Xiao L, Che HS, Li YP, Liao JT: Uterine peristalsis exerts control over fluid migration after mock embryo transfer. Hum Reprod 2014, 29:279-285.

20. Zhu L, Che HS, Xiao L, Li YP: Uterine peristalsis before embryo transfer affects the chance of clinical pregnancy in fresh and frozen-thawed embryo transfer cycles. Human Reproduction 2014, 29:1238-1243.

21. Qiong Z, Jie H, Yonggang W, Bin X, Jing Z, Yanping L: Clinical validation of pinopode as a marker of endometrial receptivity: a randomized controlled trial. Fertil Steri1 2017, 108:513-517 e512.

22. Zhang D, Ma C, Sun X, Xia H, Zhang W: S100P expression in response to sex steroids during the implantation window in human endometrium. Reprod Biol Endocrinol 2012, 10:106.

23. Eun Kwon H, Taylor HS: The role of HOX genes in human implantation. Ann N Y Acad Sci 2004, 1034:1-18.

24. Cavagna M, Mantese JC: Biomarkers of endometrial receptivity--a review. Placenta 2003, 24 Supp1 B:S39-47.

25. Dimitriadis E, White CA, Jones RL, Salamonsen LA: Cytokines, chemokines and growth factors in endometrium related to implantation. Hum Reprod Update 2005, $11: 613-630$.

26. Craciunas L, Gallos I, Chu J, Bourne T, Quenby S, Brosens JJ, Coomarasamy A: Conventional and modern markers of endometrial receptivity: a systematic review and meta-analysis. Hum Reprod Update 2019, 25:202-223.

27. Murray MJ, Meyer WR, Zaino RJ, Lessey BA, Novotny DB, Ireland K, Zeng D, Fritz MA: A critical analysis of the accuracy, reproducibility, and clinical utility of histologic endometrial dating in fertile women. Fertil Steril 2004, 81:1333-1343.

28. Quinn CE, Casper RF: Pinopodes: a questionable role in endometrial receptivity. Hum Reprod Update 2009, 15:229-236.

29. Altmae S, Esteban FJ, Stavreus-Evers A, Simon C, Giudice L, Lessey BA, Horcajadas JA, Macklon NS, D' Hooghe T, Campoy C, et al: Guidelines for the design, analysis and interpretation of 'omics' data: focus on human endometrium. Hum Reprod Update 2014, 20:12-28.

30. Horcajadas JA, Pellicer A, Simon C: Wide genomic analysis of human endometrial receptivity: new times, new opportunities. Hum Reprod Update 2007, 13:77-86.

31. Altmae S, Martinez-Conejero JA, Salumets A, Simon C, Horcajadas JA, Stavreus-Evers A: Endometrial gene expression analysis at the time of embryo implantation in women with unexplained infertility. Mol Hum Reprod 2010, $16: 178-187$.

32. Garrido-Gomez T, Ruiz-Alonso M, Blesa D, Diaz-Gimeno P, Vilella F, Simon C: Profiling the gene signature of endometrial receptivity: clinical results. Fertil Steril 2013, 99:1078-1085. 
33. Diaz-Gimeno P, Horcajadas JA, Martinez-Conejero JA, Esteban FJ, Alama P, Pellicer A, Simon C: A genomic diagnostic tool for human endometrial receptivity based on the transcriptomic signature. Fertil Steri1 2011, 95:50-60, 60 e51-15.

34. Diaz-Gimeno P, Ruiz-Alonso M, Blesa D, Bosch N, Martinez-Conejero JA, Alama P, Garrido N, Pellicer A, Simon C: The accuracy and reproducibility of the endometrial receptivity array is superior to histology as a diagnostic method for endometrial receptivity. Fertil Steril 2013, 99:508-517.

35. Ruiz-Alonso M, Blesa D, Diaz-Gimeno P, Gomez E, Fernandez-Sanchez M, Carranza F, Carrera J, Vilella F, Pellicer A, Simon C: The endometrial receptivity array for diagnosis and personalized embryo transfer as a treatment for patients with repeated implantation failure. Fertil Steril 2013, 100:818-824.

36. Simón C GC, Cabanillas S, Vladimirov I, Castillón G, Giles J, Boynukalin K, Findikli N, Bahçeci M, Ortega I, Vidal C, Funabiki M, Izquierdo A, López L, Portela S, Frantz N, Kulmann M, Taguchi S, Labarta E, Colucci F, Mackens S, Santamaría X, Muñoz E, Barrera S, García-Velasco JA, Fernández M, Ferrando M, Ruiz M, Mol BW, Valbuena D; ERA-RCT Study Consortium Group. : A 5-year multicentre randomized controlled trial comparing personalized, frozen and fresh blastocyst transfer in IVF. Reprod Biomed Online 2020, 41:14.

37. Mantione KJ, Kream RM, Kuzelova H, Ptacek R, Raboch J, Samuel JM, Stefano GB: Comparing bioinformatic gene expression profiling methods: microarray and RNA-Seq. Med Sci Monit Basic Res 2014, 20:138-142.

38. Van Royen E, Mangelschots K, De Neubourg D, Valkenburg M, Van de Meerssche M, Ryckaert G, Eestermans W, Gerris J: Characterization of a top quality embryo, a step towards single-embryo transfer. Hum Reprod 1999, 14:2345-2349.

39. Alpha Scientists in Reproductive M, Embryology ESIGo: The Istanbul consensus workshop on embryo assessment: proceedings of an expert meeting. Hum Reprod 2011, 26:1270-1283.

40. Gardner DK, Lane M, Stevens J, Schlenker T, Schoolcraft WB: Reprint of: Blastocyst score affects implantation and pregnancy outcome: towards a single blastocyst transfer. Fertil Steril 2019, 112:e81-e84.

41. DeLuca DS, Levin JZ, Sivachenko A, Fenne11 T, Nazaire MD, Williams C, Reich M, Winckler W, Getz G: RNA-SeQC: RNA-seq metrics for quality control and process optimization. Bioinformatics 2012, 28:1530-1532.

42. Dobin A, Davis CA, Schlesinger F, Drenkow J, Zaleski C, Jha S, Batut P, Chaisson M, Gingeras TR: STAR: ultrafast universal RNA-seq aligner. Bioinformatics 2013, 29:15-21.

43. Trapne11 C, Williams BA, Pertea G, Mortazavi A, Kwan G, van Baren MJ, Salzberg SL, Wold BJ, Pachter L: Transcript assembly and quantification by RNA-Seq reveals unannotated transcripts and isoform switching during cell differentiation. Nat Biotechnol 2010, 28:511-515.

44. Zegers-Hochschild F, Adamson GD, Dyer S, Racowsky C, de Mouzon J, Sokol R, Rienzi L, Sunde A, Schmidt L, Cooke ID, et al: The International Glossary on Infertility and Fertility Care, 2017 $\ddagger \S$. Human Reproduction 2017, 32:1786-1801.

45. Hu S, Yao G, Wang Y, Xu H, Ji X, He Y, Zhu Q, Chen Z, Sun Y: Transcriptomic changes 
during the pre-receptive to receptive transition in human endometrium detected by RNA-Seq. J Clin Endocrinol Metab 2014, 99:E2744-2753.

46. Altmae S, Koel M, Vosa U, Adler P, Suhorutsenko M, Laisk-Podar T, Kukushkina V, Saare M, Velthut-Meikas A, Krjutskov K, et al: Meta-signature of human endometrial receptivity: a meta-analysis and validation study of transcriptomic biomarkers. Sci Rep 2017, 7:10077.

47. Diaz-Gimeno P, Ruiz-Alonso M, Blesa D, Simon C: Transcriptomics of the human endometrium. Int J Dev Biol 2014, 58:127-137.

48. Hashimoto T, Koizumi M, Doshida M, Toya M, Sagara E, Oka N, Nakajo Y, Aono N, Igarashi H, Kyono K: Efficacy of the endometrial receptivity array for repeated implantation failure in Japan: A retrospective, two-centers study. Reprod Med Biol 2017, 16:290-296.

49. Tan J, Kan A, Hitkari J, Taylor B, Tallon N, Warraich G, Yuzpe A, Nakhuda G: The role of the endometrial receptivity array (ERA) in patients who have failed euploid embryo transfers. J Assist Reprod Genet 2018, 35:683-692.

50. Franasiak JM, Ruiz-Alonso M, Scott RT, Simon C: Both slowly developing embryos and a variable pace of luteal endometrial progression may conspire to prevent normal birth in spite of a capable embryo. Fertil Steril 2016, 105:861-866. 


\begin{tabular}{|c|c|}
\hline Characteristic & The first phase study $(n=50)$ \\
\hline Age, Mean $\pm S D, y$ & $30.9 \pm 3.89$ \\
\hline$B M I$, Mean $\pm S D, k g / m 2$ & $21.0 \pm 2.19$ \\
\hline Infertility duration, Median (IQR), y & $3(1.0-5.5)$ \\
\hline AMH, Median (IQR), ng/ml & $3.21(2.39-5.33)$ \\
\hline $\mathrm{FSH}, \mathrm{Mean} \pm \mathrm{SD}, \mathrm{mIU} / \mathrm{ml}$ & $5.63 \pm 1.15$ \\
\hline AFC, Median (IQR) & 13(9-15.75) \\
\hline Endometrial thickness, Mean $\pm S D, \mathrm{~mm}$ & $11.0 \pm 2.74$ \\
\hline \multicolumn{2}{|l|}{ Types of infertility } \\
\hline Primary infertility(n) & 6 \\
\hline Secondary infertility(n) & 44 \\
\hline \multicolumn{2}{|l|}{ IVF indication } \\
\hline Male factor(n) & 6 \\
\hline Tubal factor(n) & 44 \\
\hline
\end{tabular}

Abbreviations: rsERT, RNA-seq-based endometrial receptivity test; BMI, body mass index; AMH: antimullerian hormone; FSH: follicle-stimulating hormone; AFC: antral follicle count.

Table 2. Baseline Clinical Characteristics of the patients with RIF in experimental and control group

\begin{tabular}{|c|c|c|c|}
\hline Characteristic & Experimental $(n=56)$ & Control $(n=86)$ & $P$-value \\
\hline No. of previous failed cycles Median (IQR) & $3(2-4)$ & $3(3-4)$ & 0.462 \\
\hline Age, Mean $\pm S D, y$ & $32.71 \pm 4.14$ & $32.90 \pm 3.79$ & 0.789 \\
\hline $\mathrm{BMI}$, Mean $\pm S \mathrm{~S}, \mathrm{~kg} / \mathrm{m}^{2}$ & $21.38 \pm 2.39$ & $21.41 \pm 1.85$ & 0.926 \\
\hline Infertility duration, Mean $\pm S D, y$ & $5.18 \pm 3.42$ & $4.38 \pm 3.29$ & 0.168 \\
\hline AMH, Median (IQR), ng/ml & $2.86(1.40-5.33)$ & $4.10(2.31-6.15)$ & 0.154 \\
\hline $\mathrm{FSH}, \mathrm{Mean} \pm \mathrm{SD}, \mathrm{mlU} / \mathrm{ml}$ & $6.49 \pm 1.59$ & $6.27 \pm 1.67$ & 0.478 \\
\hline AFC, Mean $\pm S D$ & $12.55 \pm 6.91$ & $13.92 \pm 6.50$ & 0.261 \\
\hline Endometrial thickness, Mean $\pm S D, m m$ & $9.47 \pm 1.85$ & $9.26 \pm 1.42$ & 0.469 \\
\hline$P$ levels on the day of progesterone & $0.31(0.09-0.61)$ & $0.29(0.15-0.72)$ & 0.529 \\
\hline \multicolumn{4}{|l|}{ administration/LH peak, Median (IQR), ng/ml } \\
\hline \multicolumn{4}{|l|}{ Types of infertility } \\
\hline Primary infertility (n/\%) & $33(58.9 \%)$ & $41(47.7 \%)$ & \multirow{2}{*}{0.190} \\
\hline Secondary infertility (n/\%) & $23(41.1 \%)$ & $45(52.3 \%)$ & \\
\hline \multicolumn{4}{|l|}{ IVF indication } \\
\hline Male factor (n/\%) & $2(3.6 \%)$ & $3(3.5 \%)$ & \multirow{6}{*}{0.704} \\
\hline Tubal factor (n/\%) & $50(89.3 \%)$ & $82(95.3 \%)$ & \\
\hline $\operatorname{PCOS}(n / \%)$ & $6(10.7 \%)$ & $11(12.8 \%)$ & \\
\hline Diminished ovarian reserve (n/\%) & $6(10.7 \%)$ & $9(10.5 \%)$ & \\
\hline Endometriosis (n/\%) & $5(8.9 \%)$ & $3(3.5 \%)$ & \\
\hline Others (n/\%) & $2(3.6 \%)$ & $1(1.2 \%)$ & \\
\hline \multicolumn{4}{|l|}{ Sampling cycle protocol } \\
\hline Natural cycle (n/\%) & $26(46.4 \%)$ & $34(39.5 \%)$ & \multirow{2}{*}{0.416} \\
\hline HRT cycle (n/\%) & $30(53.6 \%)$ & $52(60.5 \%)$ & \\
\hline
\end{tabular}




\begin{tabular}{llll}
\hline No. of transferred embryos, Median (IQR) & $2(2-2)$ & $2(2-2)$ & 0.608 \\
Total No. of transferred embryos (n) & 90 & 158 & \\
Embryo stage & & & \\
D3 cleavage embryos (n/\%) & $51(56.7 \%)$ & $114(72.2 \%)$ & $\mathbf{0 . 0 1 3}$ \\
D5 or D6 blastocysts (n/\%) & $39(43.3 \%)$ & $44(27.8 \%)$ & \\
\hline
\end{tabular}

659

660

661

662

663

664

Abbreviations: BMI, body mass index; $\mathrm{AMH}$ : antimullerian hormone; FSH: follicle-stimulating hormone; AFC: antral follicle count; P levels: Serum endogenous progesterone level; PCOS: polycystic ovarian syndrome; HRT cycle: hormone replacement cycle.

Table 3. Pregnancy outcomes of PET in experimental group and conventional ET in control group

\begin{tabular}{|c|c|c|c|c|}
\hline & $\begin{array}{l}\text { Experimental } \\
(n=56)\end{array}$ & $\begin{array}{l}\text { Control } \\
(n=86)\end{array}$ & $\operatorname{RR}(95 \% \mathrm{Cl})$ & $P$-value \\
\hline No. of patients receiving embryo transfers $(n)$ & 48 & 86 & & \\
\hline No. of patients transferred D3 embryos (n) & 26 & 59 & & \\
\hline No. of patients who received pET (n) & 26 & / & & \\
\hline Intrauterine pregnancy $(\mathrm{n} / \%)$ & $13 / 26(50 \%)$ & $14 / 59(23.7 \%)$ & $\begin{array}{l}2.107(1.159 \\
\text { to } 3.830)\end{array}$ & 0.017 \\
\hline Live birth(n/\%) & $11 / 26(42.3 \%)$ & $13 / 59(22.0 \%)$ & $\begin{array}{l}1.92 \text { (0.995 to } \\
3.705)\end{array}$ & 0.056 \\
\hline No. of transferred D3 embryos (n) & 51 & 114 & & \\
\hline Embryos implanted (n/\%) & $16 / 51(31.4 \%)$ & $19 / 114(16.7 \%)$ & $\begin{array}{l}1.882(1.057 \\
\text { to } 3.353)\end{array}$ & 0.033 \\
\hline No. of patients transferred blastocysts ( $n$ ) & 22 & 27 & & \\
\hline No. of patients who received pET (n) & 22 & / & & \\
\hline Intrauterine pregnancy (n/\%) & $14 / 22(63.6 \%)$ & $11 / 27(40.7 \%)$ & $\begin{array}{l}1.562(0.898 \\
\text { to } 2.718)\end{array}$ & 0.111 \\
\hline Live birth(n/\%) & $13 / 22(59.1 \%)$ & $10 / 27(37.0 \%)$ & $\begin{array}{l}1.595 \quad(0.874 \\
\text { to } 2.914)\end{array}$ & 0.124 \\
\hline No. of transferred blastocysts $(n)$ & 39 & 44 & & \\
\hline Embryos implanted (n/\%) & $17 / 39(43.6 \%)$ & $12 / 44(27.3 \%)$ & $\begin{array}{l}1.598 \quad(0.877 \\
\text { to } 2.913)\end{array}$ & 0.120 \\
\hline
\end{tabular}

Abbreviations: RR, relative risk; pET, personalized embryo transfer; ET, embryo transfer;

\section{Figure legends}

Figure 1. Flow of Participants in rsERT-guided pET Trial (The second phase of the current study). Figure 2. Hierarchical clustering of the RNA expression data from 50 individuals; three samples per individual were obtained, one at each ER stage.

Figure 3. Establishment and validation of the RNA-seq-based endometrial receptivity test (rsERT). A. Linear discriminant analysis (LDA) of endometrial receptivity conditions based on selected predictive markers; $\mathrm{B}$. ROC curves generated by 100 random splits into a training set and a test set. 
Figure 4. Performance of WOI time prediction model. (A) Comparison of predicted WOI time in different receptivity stages. (B) Hour difference between predicted and observed WOI time. (C)

679 Cumulative distribution of WOI time prediction deviation from observed ones, showing $94 \%$ and

$68085 \%$ of cases with the deviation of less than 24 and 12 hours between predictions and the 681 expected ones

682

683

684

685 


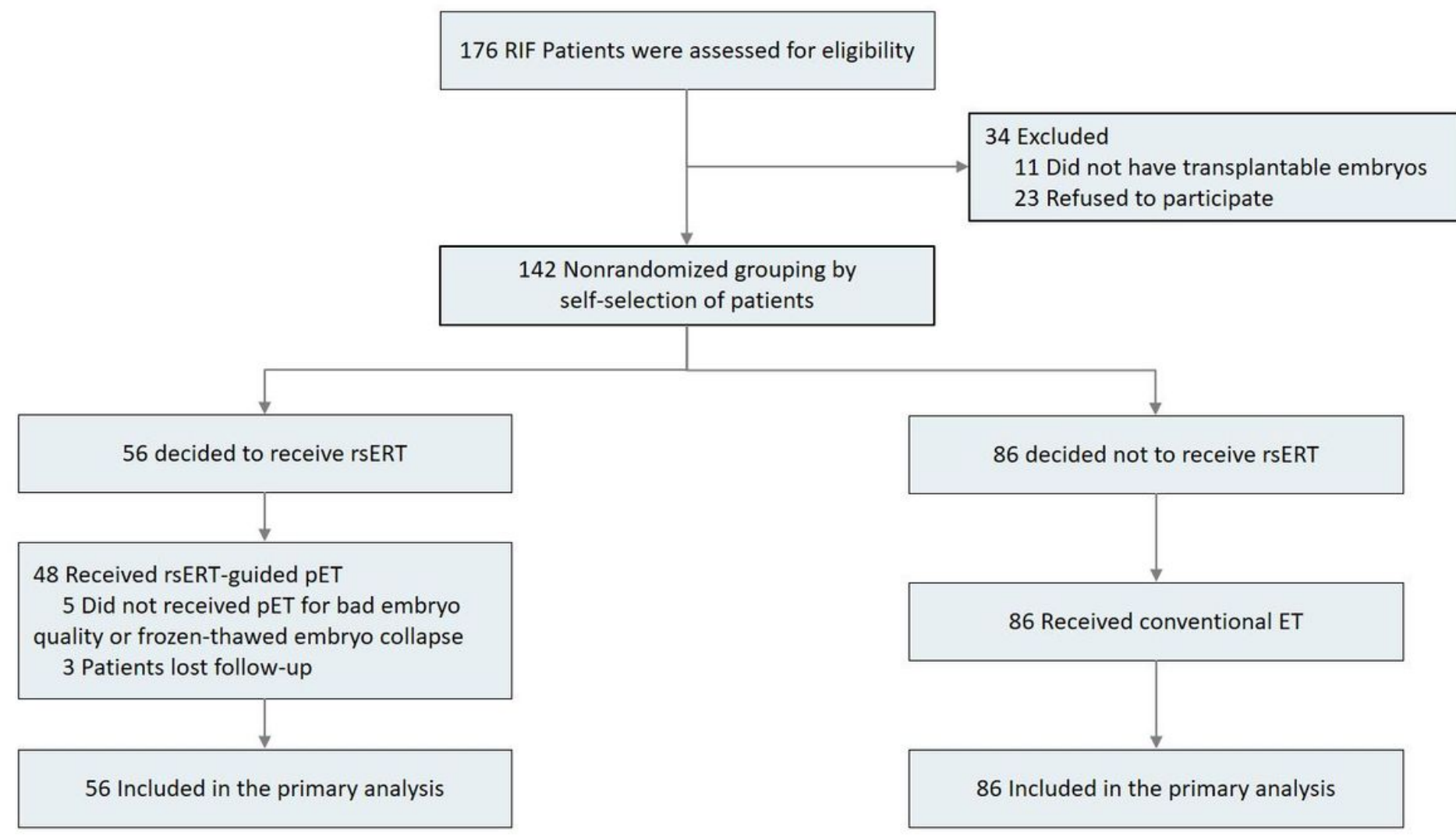

\section{Figure 1}

Flow of Participants in rsERT-guided pET Trial (The second phase of the current study). 


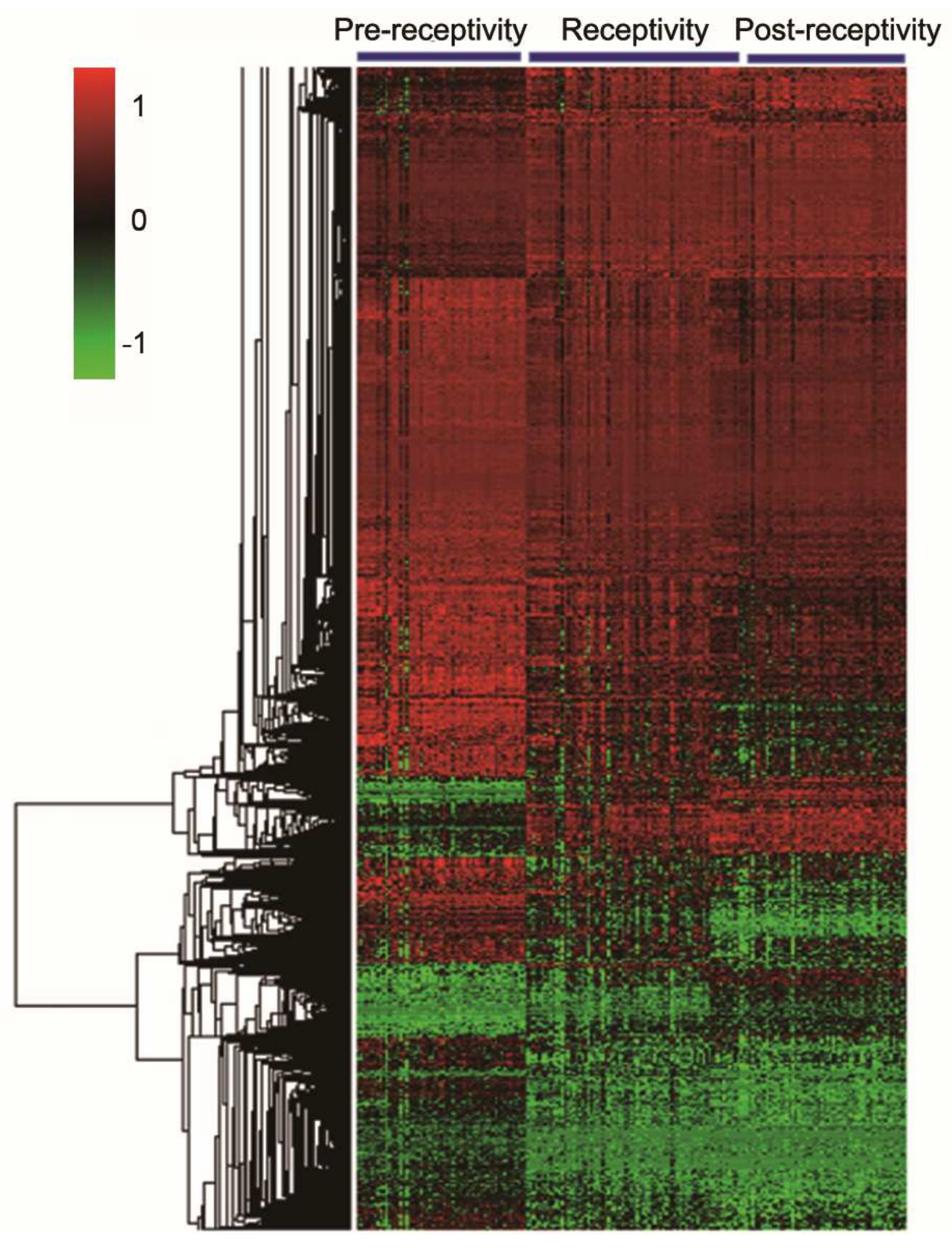

Figure 2

Hierarchical clustering of the RNA expression data from 50 individuals; three samples per individual were obtained, one at each ER stage. 
A.

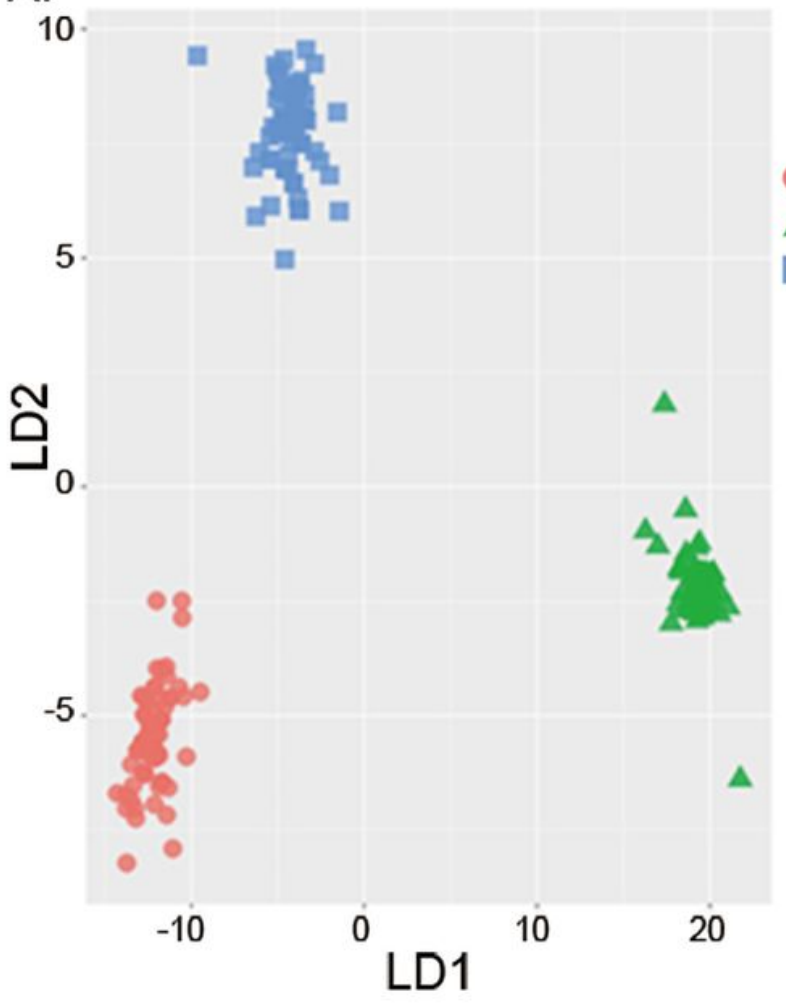

B.

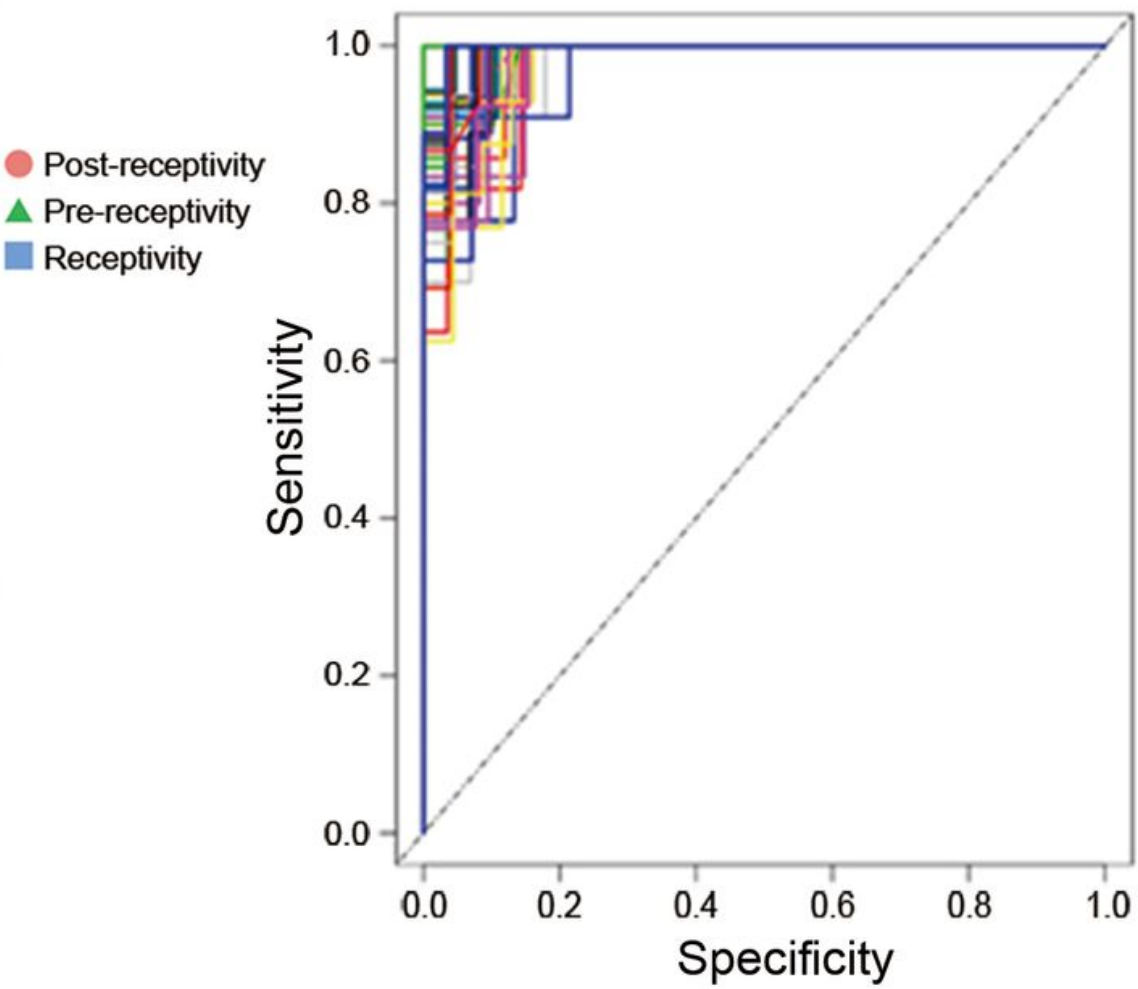

Figure 3

Establishment and validation of the RNA-seq-based endometrial receptivity test (rsERT). A. Linear discriminant analysis (LDA) of endometrial receptivity conditions based on selected predictive markers; $B$. ROC curves generated by 100 random splits into a training set and a test set.
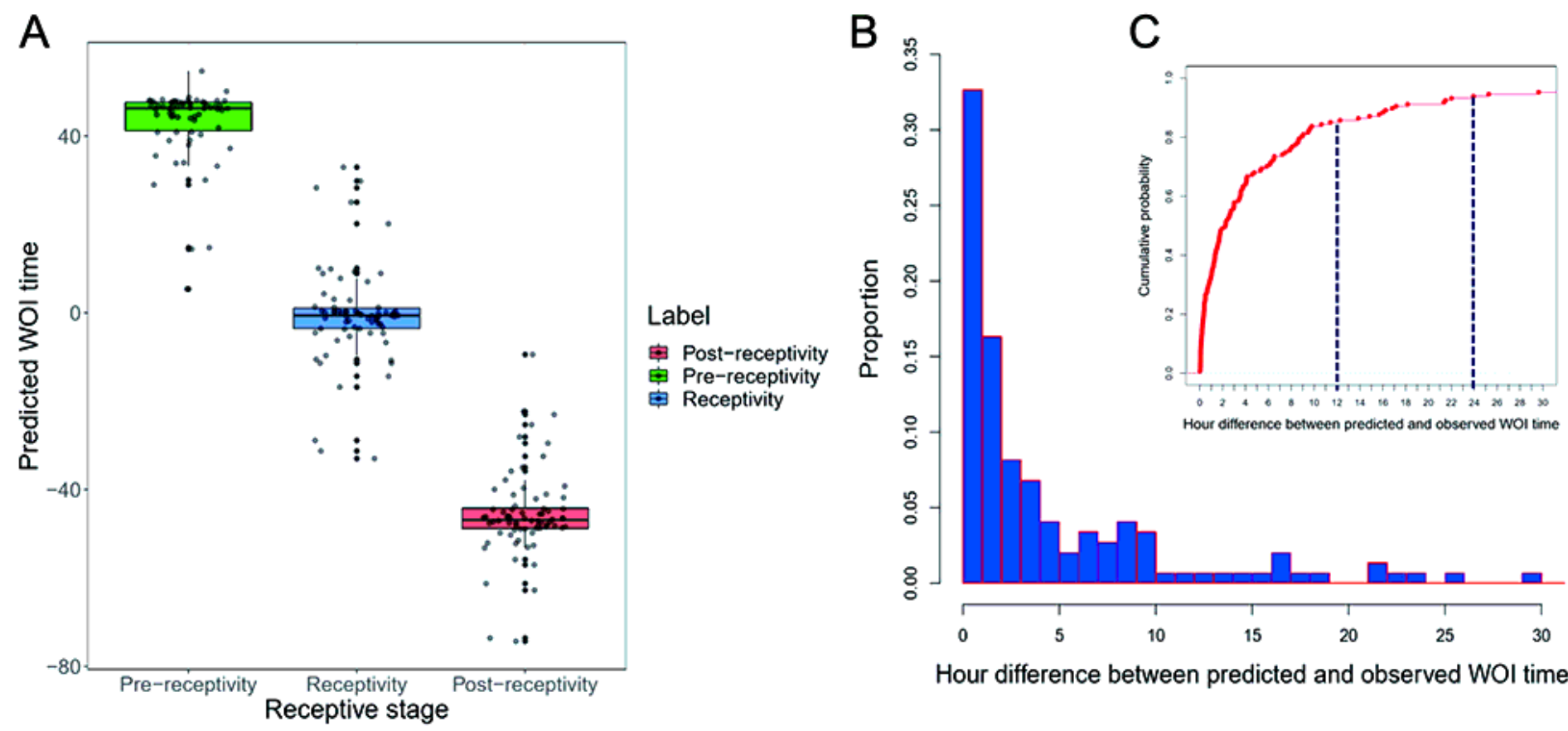

Hour difference between predicted and observed WOI time

Figure 4 
Performance of WOI time prediction model. (A) Comparison of predicted WOI time in different receptivity stages. (B) Hour difference between predicted and observed WOI time. (C) Cumulative distribution of WOI time prediction deviation from observed ones, showing $94 \%$ and $85 \%$ of cases with the deviation of less than 24 and 12 hours between predictions and the expected ones

\section{Supplementary Files}

This is a list of supplementary files associated with this preprint. Click to download.

- SupplementaryS1GOandKEGGannotation.xls

- SupplementaryS2Listofpredictivemarkers.xls 